\title{
Research and Construction of Intelligent Service Platform for Aquaculture
}

\author{
Feng Yan $^{1, a)}$,Wang Xinan ${ }^{1, b)}$ and Li Haitao ${ }^{1, c)}$ \\ ${ }^{1}$ School of Information Science and Technology, Qingdao University of Science and Technology, \\ Qingdao ShanDong 266000, China. \\ a)fywmh@163.com \\ b) $370017466 @ q q . c o m$ \\ c)32891741@qq.com
}

Keywords: Construction, Intelligent service, Platform, Aquaculture

\begin{abstract}
Intelligent aquaculture, refers to the engineering technology, machinery and equipment, monitoring instruments, wireless sensor, management software and large data, cloud computing, artificial intelligence and other modern technical means for fishery production, to achieve high density, high output value, high efficiency Standardized farming model. Intelligent aquaculture cloud service platform, mainly from the data collection, data processing, data application of three aspects of analysis and design, auxiliary farming enterprises to carry out breeding decision-making, improve the level of intelligence farming. The use of industrialization and intelligence in the field of aquaculture in the vertical diffusion and deep integration, to explore the modern farming model, enhance the contribution of intelligent technology in the development of fishery economy.
\end{abstract}

\section{INTRODUCTION}

China is a large aquaculture country, aquaculture is in the stage of industrial transformation, from relying on the experience, extensive management of the traditional farming model, to the modernization, information technology, intelligent new farming model change ${ }^{[1]}$. The former to the huge aquaculture industry to bring great risk of farming. The wisdom of the model will use the modern advanced technology to avoid these shortcomings, accurate and reliable, scientific and rational management of aquatic aquaculture process to ensure that aquatic products production and quality.

\section{PLATFORM OVERVIEW}

Intelligent aquaculture cloud service platform is based on data collection, through data processing, and ultimately applied to a set of intelligent aquaculture information platform software. Platform with the current advanced software engineering ideas, mobile Internet thinking, methods and tools, using a variety of modern information technology. Platform for aquaculture management, management, service work to provide a basis for decision-making.

\subsection{Platform functional architecture}

The platform's functional architecture mainly includes three parts, data collection, data processing, data application ${ }^{[2]}$. Data acquisition is the basis of the entire platform, data processing and data applications are dependent on the basic data; data processing refers to the collection of data for sorting, sorting, analysis, display, and better serve the data; Application refers to the basic data based on the collection, after processing the correct data, the analysis and research to help users make decisions, and then counteract the aquaculture.

\subsection{Platform function analysis}

Platform from the data acquisition, data processing, data application of three aspects of analysis and construction.

\subsubsection{Data collection}

The acquisition of data includes the acquisition of empirical knowledge data, the acquisition of 
microscopic data and the acquisition of macroscopic data.

(1) Experience knowledge refers to the breeding experience, the latest breeding dynamics, professional farming technology, mature farming model. Access to this knowledge, through artificial data mining processing, so as to help aquaculture related decision-making.

(2)The acquisition of microscopic data mainly includes the acquisition of water quality parameters, the acquisition of meteorological data in the breeding area, and the acquisition of animal video images. Microscopic data is the basis of the core functions of the system, providing data for breeding decisions and so on.

(3) Macro data acquisition refers to the aquaculture area of the water environment information, spatial information collection.

\subsubsection{Data processing}

Data processing refers to the collection of data to sort, classification, mining, entry into the database, or in the system interface to display, user-friendly query, operation.

(1) Experience knowledge data processing, the classification of breeding experience, sorting, storage process, the establishment of the corresponding knowledge base.

(2) Water quality monitoring is an important part of aquaculture, and the quality of water environment determines the quality of aquatic products. Water quality monitoring data processing is divided into the following aspects:

A. Quality control of water quality monitoring data;

B. Real-time display of water quality data;

C. Water quality cycle trends show.

(3) Underwater video surveillance data, mainly underwater shooting video images, real-time transmission to the cloud, through the PC, mobile phone real-time view, analysis, understanding of aquatic product growth status.

(4) The spatial data of the waters include the water quality and spatial changes of the whole waters. Through the data mining technology, analysis and evaluation of water quality, the degree of water quality and other data, to the user with an intuitive, clear display. From the macro perspective to reveal the dynamic changes in its farming area.

\subsubsection{Data application}

(1) Intelligent monitoring. Based on the intelligent network sensor to collect the monitoring data, 24 hours of dynamic detection and automatic control. Water quality monitoring and control process shown in Figure 1.

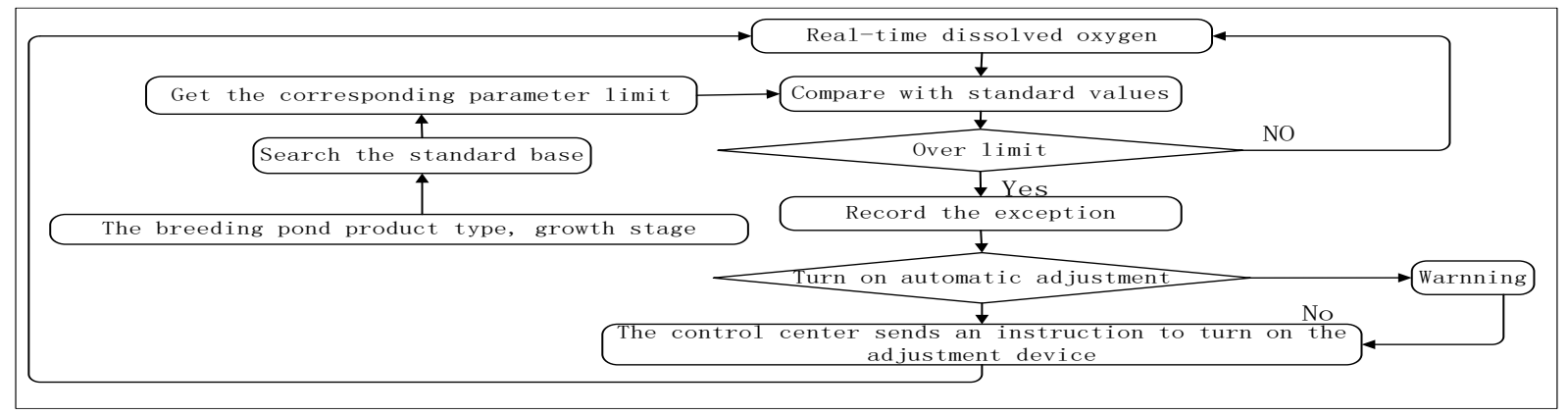

FIGURE 1. Water quality monitoring and intelligent control flow chart

(2) disease diagnosis and prevention. The system is mainly developed from the following three aspects.

A. Build a disease diagnosis library;

B. Establish expert remote diagnostic mechanism ;

C. Establish a platform for the diagnosis and prevention of scientific knowledge of aquatic products.

(3) Aquatic product model establishment. The water quality prediction model and the prediction model of aquatic product growth were established. Example Figure 2, Figure 3. 


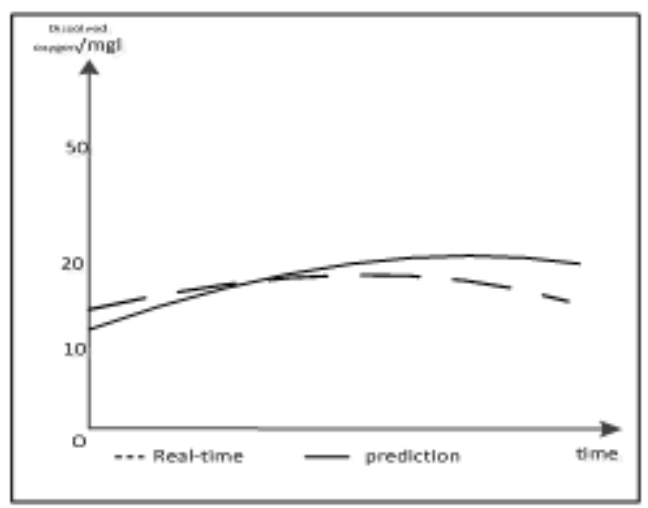

FIGURE 2.

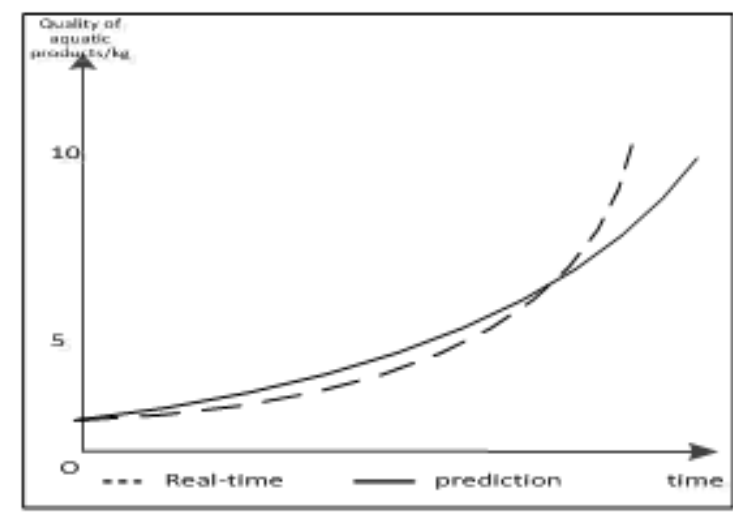

FIGURE 3

\section{PLATFORM CONSTRUCTION}

The platform is divided into three subsystems for design, basic data application, water quality on-line monitoring analysis and forecasting, aquatic product growth prediction. Platform mainly includes data center software, Web client, mobile APP client. The network topology of the platform is shown in Figure 4.

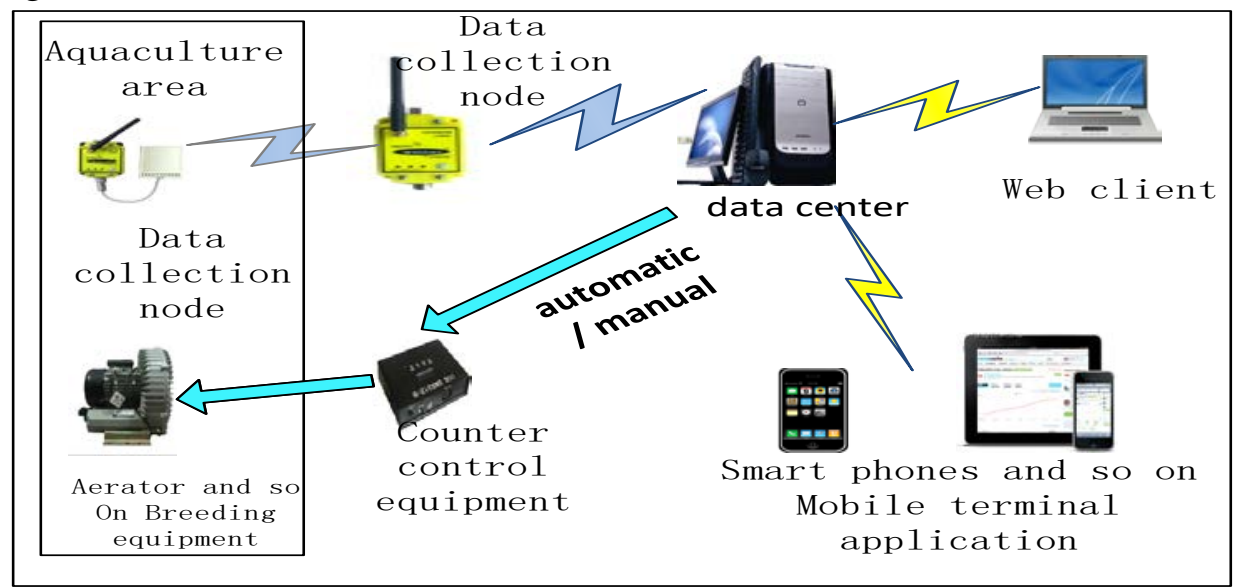

FIGURE 4. Platform Network Topology

A. Basic data application design. The application of basic data refers to the acquisition of experience knowledge, micro data, macro data, through computer technology management.

B. On line monitoring and analysis of water quality. Water quality analysis and prediction is based on the collected data, and real-time data analysis, through the water quality prediction model, water quality data prediction, analysis and evaluation.

C. Aquatic product growth prediction design. The growth trend of each aquatic product was predicted by establishing the production prediction model for each aquatic product. The user or the enterprise through the terminal equipment, to see the actual growth curve and prediction curve of aquatic products.

D. Database design. The central database of this platform uses Oracle database. The central database includes industry dynamic table, breeding technology table, disease prevention and control technology, quality standard database and expert information table, quality information table, video information table, water table, water product information product growth data table, feed information table, system log table etc..

\section{PLATFORM KEY TECHNOLOGY}

In the process of system construction, the key technologies of data collection, data processing and data application in the process of aquaculture intelligence are studied. Including data acquisition technology, data processing and application technology. Specifically divided into: 
(1) knowledge mining technology

(2) intelligent sensor network technology

(3) GIS and space remote sensing technology

(4) Data repair technology

(5) Intelligent control technology

\section{CONCLUSION}

Through the development of intelligent aquaculture service platform and the research of its key technology, it is deeply realized that the intelligentization of aquaculture has become an important connotation and support of modern fishery, which can promote the development of aquaculture and the establishment of fishery informatization system , And enhance the farmers to participate in market capacity and quality of farming. Platform has a complete cloud service system to meet the various needs of fishermen, easy to expand, but there are also deficiencies, because the lack of theoretical support for the establishment of the model, this link is relatively weak. The future will strengthen this research.

\section{ACKNOWLEDGMENTS}

This work is supported by the Natural Science Foundation of Shandong Province in China (ZR2013FL014) and the Science and Technology Program of Shandong University of China(J13LN73).

\section{REFERENCES}

[1]. Liu Jinquan, Construction of soft and hard combination of aquaculture networking solutions, Internet of things technology, 2013, sixth, 10-11.

[2]. Hu Jinyou, Wang Jingjie, Zhang Xiaotong, etc. Current status and trends of key technologies in a) aquaculture information technology, Journal of Agricultural Mechanics, 2015,46 (7): 251263.

[3]. Liu Shuangyin,Based on the computational intelligence of aquaculture water quality prediction and early

a) warning methods, doctoral thesis, 2014.

[4]. $\mathrm{Xu} \mathrm{Su,Application} \mathrm{of} \mathrm{computer} \mathrm{vision} \mathrm{in} \mathrm{aquaculture} \mathrm{and} \mathrm{production,} \mathrm{Journal} \mathrm{of} \mathrm{Tianjin}$ Agricultural College, 2014,21 (4): 43-46.

[5]. Liu Guang-ming,On the Construction of Intensive Culture Information Platform Based on Cloud Computing, Jiangsu Agricultural Science, 2013,41 (12): 248-251.

[6]. Cai Wengui.Application of Geographic Information System in Seawater Aquaculture Environment Research, Taiwan Strait, 2004,22 (3): 253-258.

[7]. Xiao Le, China's "Internet + aquaculture" development status and path of the study. Fishery modernization, 2016,43 (3): 7-11.

[8]. Jia Haitian, Wang Rongxiang, Sun Shun. Development and application of breeding platform based on mobile Internet, computer programming skills and maintenance, 2014,61-62.

[9]. Li Daoliang, Fu Zetian.Design and preliminary implementation of intelligent aquaculture information system, Journal of Agricultural Engineering, 2000,16 (4): 135-138. 